\title{
PERFIL DAS VÍTIMAS DE AFECÇÕES NEUROLÓGICAS ATENDIDAS POR UM SERVIÇO PRÉ-HOSPITALAR MÓVEL DE URGÊNCIA
}

\author{
Sabrina Daiane Gurgel Sarmento ${ }^{1}$, Rodrigo Assis Neves Dantas ${ }^{2}$, Daniele Vieira Dantas ${ }^{3}$, Sara Porfírio de \\ Oliveira $^{1}$, Lívia Maria Nunes Henriques ${ }^{1}$, Izabelle Bezerra Costa ${ }^{1}$
}

\begin{abstract}
RESUMO: Este artigo tem por objetivo caracterizar o perfil epidemiológico das vítimas de afecções neurológicas atendidas por um Serviço de Atendimento Móvel de Urgência. Trata-se de estudo exploratório, descritivo e de abordagem quantitativa dos dados. A coleta foi realizada no período de janeiro a junho de 2016, em um hospital referência em atendimentos de urgência e emergência do estado do Rio Grande do Norte. Foram analisadas 73 ocorrências neurológicas, 38 (52,1\%) do sexo masculino, 43 (58,9\%) entre a faixa etária de 68 a 101 anos. Quanto à característica da afecção neurológica, 59 (80,8\%) sofreram Acidente Vascular Encefálico, oito (11\%) crise convulsiva, três $(4,1 \%)$ lombalgia associada a lesão medular e três $(4,1 \%)$ eram de outra natureza neurológica. Quanto ao tempo resposta para o atendimento, a média foi de 31 minutos. O perfil epidemiológico identificado foi de homens idosos, que sofreram Acidente Vascular Encefálico e que não receberam atendimento no tempo resposta adequado.
\end{abstract}

DESCRITORES: Serviços médicos de emergência; Doenças do sistema nervoso; Acidente vascular cerebral; Epilepsia; Dor lombar.

\section{PROFILE OF INDIVIDUALS WITH NEUROLOGICAL DISORDERS ASSISTED BY A PREHOSPITAL MOBILE EMERGENCY CARE SERVICE}

\begin{abstract}
This article aims to characterize the epidemiological profile of individuals with neurological disorders assisted by a prehospital mobile emergency care service. Exploratory and descriptive study with quantitative approach. Data was collected from January to June 2016, in an emergency and urgent care referral center in the state of Rio Grande do Norte. Seventy-three (73) neurological disorders were analyzed: $38(52.1 \%)$ patients were male individuals, $43(58.9 \%)$ were aged between 68 and 101 years. Regarding the characteristics of the neurological condition, $59(80.8 \%)$ patients had a cerebrovascular accident (CVA), eight (11\%) had seizures, three $(4.1 \%)$ had low back pain related to spinal cord trauma and three $(4.1 \%)$ patients had other types of neurological conditions. As for the response time to care, the average time was 31 minutes. The epidemiological profile identified was elderly men who had CVA (stroke) and whose care needs were not responded within an appropriate time.
\end{abstract}

DESCRIPTORS: Emergency medical services; Nervous system disorders; Cerebrovascular Accident (stroke); Epilepsy; Low back pain.

\section{PERFIL DE VÍCTIMAS DE AFECCIONES NEUROLÓGICAS ATENDIDAS POR SERVICIO PREHOSPITALARIO MÓVIL DE URGENCIA}

RESUMEN: Artículo que objetiva caracterizar el perfil epidemiológico de víctimas de afecciones neurológicas atendidas por un Servicio de Atención Móvil de Urgencia. Estudio exploratorio, descriptivo, de abordaje cuantitativo de datos. La recolección se realizó de enero a junio de 2016, en hospital de referencia en atención de urgencias y emergencias del estado de Rio Grande do Norte. Fueron analizados 73 sucesos neurológicos, 38 (52,1\%) en pacientes masculinos; $43(58,9 \%)$ pertenecientes a faja etaria de 68 a 101 años. Respecto a características de la afección, 59 (80,8\%) sufrieron Accidente Cerebrovascular, 8 (11\%) crisis convulsiva, 3 (4,1\%) lumbalgia asociada a lesión medular, y $3(4,1 \%)$ fueron de otra naturaleza neurológica. Acerca del tiempo de respuesta, el promedio fue de 31 minutos. El perfil epidemiológico identificado fue de hombres ancianos, que sufrieron Accidente Cerebrovascular y que no recibieron atención en el tiempo de respuesta adecuado.

DESCRIPTORES: Servicios Médicos de Urgencia; Enfermedades del Sistema Nervioso; Accidente Cerebrovascular; Epilepsia; Dolor de la Región Lumbar.

${ }^{1}$ Discente de Enfermagem. Universidade Federal do Rio Grande do Norte. Natal, RN, Brasil.

Enfermeiro. Doutor em Ciências da Saúde. Docente de Enfermagem da Universidade Federal do Rio Grande do Norte. Natal, RN, Brasil.

${ }^{3}$ Enfermeira. Pós-doutoranda em Enfermagem. Docente de Enfermagem da Universidade Federal do Rio Grande do Norte. Natal, RN, Brasil.

Autor Correspondente:

Sabrina Daiane Gurgel Sarmento

Universidade Federal do Rio Grande do Norte

Rua dos Tororos, 420 - 59054550 - Natal, RN, Brasil

E-mail: sabrinagurgelsarm@gmail.com
Recebido: $12 / 12 / 2016$

Finalizado: 19/04/2017 


\section{INTRODUÇÃO}

Os dados epidemiológicos brasileiros sofreram transformação refletindo em mudanças no perfil de adoecimento e saúde da população. As doenças infectocontagiosas e parasitárias deixaram de ser as principais causas de morte no Brasil, e deram espaço, na atualidade, às doenças crônicas não transmissíveis e às causas externas, provocando alteração nos atendimentos dos serviços de urgência e emergência ${ }^{(1-2)}$.

Diante do exposto, os serviços de saúde necessitaram atender a nova demanda. Para tanto, o Serviço de Atendimento Móvel de Urgência (SAMU) foi criado em 2003 através da Portaria n. 1863/ $\mathrm{GM}^{(3)}$, instituído pela Política Nacional de Atenção às Urgências. O SAMU tem como objetivo promover atendimento imediato e resolutivo no transporte para um serviço de saúde. Em 2014, no Brasil, o serviço atendeu $75 \%$ da população brasileira, distribuído em 2.921 municípios ${ }^{(4)}$.

Em um estudo americano, realizado em hospitais de emergências, as queixas neurológicas correspondiam a $4,7 \%$ dos atendimentos ${ }^{(5)}$. No Brasil, um estudo demonstrou que as emergências neurológicas mais comuns são: acidente vascular encefálico, epilepsia, cefaleia e outros sintomas secundários à condição clínica. Este mesmo estudo mostrou ainda que $11 \%$ dos pacientes atendidos na emergência precisaram de uma avaliação neurológica ${ }^{(6)}$.

A demora no atendimento, bem como a espera para realização de exames, representam grande fator de risco para complicação em situações neurológicas. Portanto, a rapidez no atendimento e reconhecimento dos sintomas pelos profissionais do SAMU como também uma transferência para um serviço especializado de forma adequada e rápida é de grande relevância ${ }^{(7)}$.

Este estudo possibilitará o conhecimento do perfil dos usuários que procuram o serviço préhospitalar móvel de urgência, colaborando para elaboração de estratégias mais efetivas para realização do atendimento, como também servirá de alerta para os serviços de saúde e gestores quanto à criação de medidas de prevenção e controle da população em risco a esse agravo à saúde.

Diante do mencionado, percebe-se a necessidade de conhecer as principais afecções neurológicas atendidas pelo SAMU. Para tanto, objetivou-se no presente estudo: caracterizar o perfil epidemiológico das vítimas de afecções neurológicas atendidas por um Serviço de Atendimento Móvel de Urgência 192 Rio Grande do Norte (SAMU 192 RN).

\section{MÉTODO}

Trata-se de um estudo exploratório, descritivo e de abordagem quantitativa dos dados, realizado no pronto socorro de um complexo hospitalar que é a referência nos atendimentos de urgência e emergência do estado do Rio Grande do Norte (RN).

A amostra, por conveniência, foi de 73 vítimas de afecções neurológicas ${ }^{(8)}$ atendidas pelo SAMU 192 $\mathrm{RN}$, após serem estabilizadas e transportadas para o referido hospital.

Os critérios de inclusão para participação nessa pesquisa foram: ter idade igual ou superior a 18 anos; estar consciente ou com a presença de algum acompanhante legalmente responsável, no caso de instabilidade hemodinâmica. Excluíram-se as vítimas que tinham lesões neurológicas ocasionadas por traumas.

O procedimento de coleta dos dados ocorreu no período de janeiro a junho de 2016, através da aplicação de um instrumento estruturado, composto por questões que abordavam os dados sociodemográficos (idade, sexo, grau de escolaridade, renda e ocupação), agravo neurológico sofrido, horário de atendimento, tempo resposta do atendimento e o tipo de veículo que realizou o transporte da vítima.

As vítimas ou responsáveis eram orientadas quanto ao propósito da pesquisa, riscos e benefícios. Os que concordavam em participar, assinavam o Termo de Consentimento Livre e Esclarecidos (TCLE) (9). 
Os dados foram digitados e analisados por meio de estatística descritiva, utilizando planilha no Microsoft Excel (Office 2016) e apresentados em formas de tabelas.

O presente estudo cumpre todos os aspectos éticos regidos pela Resolução $n^{\circ} 466$, de 12 de dezembro de $2012^{(9)}$, do Conselho Nacional de Saúde, que trata das normas para pesquisa envolvendo seres humanos. Levando em consideração a resolução, submeteu-se à análise do comitê de ética em pesquisa do Hospital Universitário Onofre Lopes (HUOL), de Natal/RN, obtendo parecer favorável, sob parecer n. 437/2010.

\section{RESULTADOS}

Em relação às características das afecções neurológicas, observou-se que 59 (80,8\%) deram entrada na urgência por Acidente Vascular Encefálico (AVE), oito (11\%) por crise convulsiva, três $(4,1 \%)$ por lombalgia associada a lesão medular, e três $(4,1 \%)$ eram de outras naturezas neurológicas, como mostra a Tabela 1.

Tabela 1 - Caracterização das vítimas de afecções neurológicas atendidas pelo SAMU 192 quanto ao sexo, idade e grau de escolaridade. Natal, RN, Brasil, 2016

\begin{tabular}{|c|c|c|c|c|c|}
\hline & AVE* & $\begin{array}{c}\begin{array}{c}\text { Lombalgias/ } \\
\text { Lesão Medular }\end{array} \\
\end{array}$ & $\begin{array}{c}\text { Crise } \\
\text { Convulsiva }\end{array}$ & Outros** & Total \\
\hline Variáveis & n (\%) & n (\%) & n (\%) & n (\%) & n (\%) \\
\hline \multicolumn{6}{|l|}{ Sexo } \\
\hline Masculino & $32(54,2)$ & $1(33,3)$ & $5(62,5)$ & $0(0)$ & $38(52,1)$ \\
\hline Feminino & $27(45,8)$ & $2(66,7)$ & $3(37,5)$ & $3(100)$ & $35(47,9)$ \\
\hline \multicolumn{6}{|l|}{ Faixa etária } \\
\hline 18 a 35 anos & $1(1,7)$ & $1(33,3)$ & $1(12,5)$ & $1(33,3)$ & $4(5,5)$ \\
\hline 36 a 67 anos & $19(32,2)$ & $3(66,7)$ & $5(62,5)$ & $0(0)$ & $26(35,6)$ \\
\hline 68 a 101 anos & $39(66,1)$ & $0(0)$ & $2(25)$ & $2(66,7)$ & $43(58,9)$ \\
\hline \multicolumn{6}{|l|}{ Grau de escolaridade } \\
\hline Não alfabetizado & $22(37,3)$ & $0(0)$ & $0(0)$ & $1(33,3)$ & $23(31,5)$ \\
\hline Ensino fundamental I & $18(30,5)$ & $0(0)$ & $3(37,5)$ & $0(0)$ & $21(28,8)$ \\
\hline Ensino fundamental II & $11(18,6)$ & $1(33,3)$ & $2(25)$ & $0(0)$ & $14(19,2)$ \\
\hline Ensino médio & $7(11,9)$ & $2(66,7)$ & $3(37,5)$ & $2(66,7)$ & $14(19,2)$ \\
\hline Ensino superior & $0(0)$ & $0(0)$ & $0(0)$ & $0(0)$ & $0(0)$ \\
\hline Pós-graduação & $1(1,7)$ & $0(0)$ & $0(0)$ & $0(0)$ & $1(1,4)$ \\
\hline Total & $59(80,8)$ & $3(4,1)$ & $8(11)$ & $3(4,1)$ & $73(100)$ \\
\hline
\end{tabular}

*AVE: Acidente Vascular Encefálico

**Outros: síncopes e parestesias inespecíficas.

De acordo com o total de ocorrências, 38 (52,1\%) eram do sexo masculino, 43 (58,9\%) estavam na faixa etária entre 68 a 101 anos e $23(31,5 \%)$ eram não alfabetizados. Outro dado a ser considerada é a renda mensal dos participantes, 51 (69,9\%) recebiam de um a dois salários mínimos (SM); 19 (26,0\%), menos que um SM e três $(4,1 \%)$, de três a cinco SM.

Na Tabela 2, observa-se que 48 (65,8\%) são do lar ou aposentados(as), em seguida, os trabalhadores do setor de comércio e outros serviços com 11 (15,1\%), e o desemprego quatro (5,5\%). 
Tabela 2 - Caracterização das vítimas de afecções neurológicas atendidas pelo SAMU 192 quanto à ocupação. Natal, RN, Brasil, 2016

\begin{tabular}{lccccc} 
OCUPAÇÃO & AVE* & $\begin{array}{c}\text { Lombalgia/ } \\
\text { Lesão Medular }\end{array}$ & $\begin{array}{c}\text { Crise } \\
\text { Convulsiva }\end{array}$ & Outros** & Total \\
\hline Trabalhador do campo & $\mathbf{n ~ ( \% )}$ & $\mathbf{n ~ ( \% )}$ & $\mathbf{n ~ ( \% )}$ & $\mathbf{n ~ ( \% )}$ & $\mathbf{n}(\%)$ \\
\hline Desempregado & $3(5,1)$ & $0(0)$ & $0(0)$ & $0(0)$ & $3(4,1)$ \\
\hline Construção civil & $3(5,1)$ & $0(0)$ & $1(12,5)$ & $0(0)$ & $4(5,5)$ \\
\hline Comércio e outros serviços & $1(1,7)$ & $1(33,3)$ & $1(12,5)$ & $0(0)$ & $3(4,1)$ \\
\hline Profissional liberal & $6(1,7)$ & $2(66,7)$ & $3(37,5)$ & $0(0)$ & $11(15,1)$ \\
\hline Autônomo & $1(1,7)$ & $0(0)$ & $0(0)$ & $0(0)$ & $1(1,4)$ \\
\hline Do lar ou aposentado(a) & $2(3,4)$ & $0(0)$ & $0(0)$ & $1(33,3)$ & $3(4,1)$ \\
\hline Total & $43(72,9)$ & $0(0)$ & $3(37,5)$ & $2(66,7)$ & $48(65,8)$ \\
\hline
\end{tabular}

*AVE: Acidente Vascular Encefálico

**Outros: síncopes e parestesias inespecífica.

Tratando-se do turno de atendimento realizado pelo SAMU (Tabela 3), o turno matutino (00 hora 01 minuto às 11 horas59minutos) representou 37 (50,7\%) e o turno vespertino (12 horas às 18horas59minutos) totalizou 29 (39,7\%).

Tabela 3 - Caracterização das vítimas de afecções neurológicas atendidas pelo SAMU 192 quanto ao horário de atendimento e tipo de transporte utilizado. Natal, RN, Brasil, 2016

\begin{tabular}{lccccc} 
Variáveis & AVE* & $\begin{array}{c}\text { Lombalgia/ } \\
\text { Lesão Medular }\end{array}$ & $\begin{array}{c}\text { Crise } \\
\text { Convulsiva }\end{array}$ & Outros** & Total \\
\cline { 2 - 6 } & $\mathbf{n ~ ( \% )}$ & $\mathbf{n ~ ( \% )}$ & $\mathbf{n ~ ( \% )}$ & $\mathbf{n ~ ( \% )}$ & $\mathbf{n ~ ( \% )}$ \\
\hline HORÁRIO DO ATENDIMENTO & & & & & \\
\hline Manhã (00:01 às 11h59min) & $30(50,8)$ & $3(100)$ & $1(37,5)$ & $1(33,3)$ & $37(50,7)$ \\
\hline Tarde (12 às 18h59min) & $24(40,7)$ & $0(0)$ & $4(50)$ & $1(33,3)$ & $29(39,7)$ \\
\hline Noite (19 às 24h) & $5(8,5)$ & $0(0)$ & $1(12,5)$ & $1(33,3)$ & $7(9,6)$ \\
\hline TIPO DE VEÍCULO DE TRANSPORTE & & & & & \\
\hline Unidade de Suporte Básico (UBS) & $37(62,7)$ & $3(100)$ & $5(62,5)$ & $2(66,7)$ & $47(64,4)$ \\
\hline Unidade de Suporte Avançado (USA) & $20(33,9)$ & $0(0)$ & $3(37,5)$ & $1(33,3)$ & $24(32,9)$ \\
\hline Unidade de Resgate (UR) & $1(1,7)$ & $0(0)$ & $0(0)$ & $0(0)$ & $1(1,4)$ \\
\hline Helicóptero & $1(1,7)$ & $0(0)$ & $0(0)$ & $0(0)$ & $1(1,4)$ \\
\hline Total & $59(80,8)$ & $3(4,1)$ & $8(11,0)$ & $3(4,1)$ & $73(100)$
\end{tabular}

*AVE: Acidente Vascular Encefálico

**Outros: síncopes e parestesias inespecíficas.

O dado referente ao tipo de veículo utilizado no atendimento dos pacientes, a Tabela 3 revelou que a Unidade de Suporte Básico (USB) atendeu a 47 (64,4\%) das ocorrências. A Unidade de Suporte Avançado (USA), que presta assistência aos pacientes mais graves, foi utilizada em 24 (32,9\%) atendimentos.

Quanto ao tempo resposta, que corresponde ao tempo gasto entre o acionamento do SAMU 192 até a chegada da ambulância ao local da ocorrência, a média foi de 31 minutos, sendo o menor tempo de 10 minutos e o maior, 90 minutos. 


\section{- DISCUSSÃO}

Nesta análise descritiva do perfil epidemiológico das afecções neurológicas atendidas pelo SAMU 192 RN, o AVE e a crise convulsiva foram os principais agravos à saúde desta natureza. Estudo brasileiro encontrou dado semelhante ${ }^{(6)}$. Um outro diagnóstico observado foi a lombalgia associada à lesão medular. Pesquisa realizada nos Estados Unidos detectou o mesmo diagnóstico em 12 (8,3\%) das vítimas atendidas ${ }^{(7)}$.

Esses dados fornecem um diagnóstico da necessidade de saúde da população atendida pelo serviço, dessa forma servirá para criar estratégias eficazes e de melhoria tanto no que concerne o atendimento em si, como também a estrutura, recursos humanos e de equipamento especializado para responder às necessidades do agravo.

Nesse estudo houve predomínio do sexo masculino. Ressalta-se que homens ainda são ancorados aos atributos de força e virilidade, e por outro lado demonstram fraqueza e fragilidade no cuidado com a saúde. Diante disso, explica a persistência de fatores de riscos e vulnerabilidade a agravos neurológicos ${ }^{(10-12)}$.

Em um estudo realizado em Porto Alegre-RS, a faixa etária de 41 a 60 anos concentrou o maior número de atendimentos realizados pelo SAMU aos agravos neurológicos. Em contrapartida, na presente pesquisa a idade de 68 a 101 anos foi mais frequente, já a crise convulsiva compartilha de uma faixa etária semelhante ao estudo referido, apresentando a idade de 40 a 60 anos $^{(13)}$. Percebe-se que a população idosa foi acometida em maior número nesse estudo. O envelhecimento da população brasileira é uma realidade que requer mudança no setor da saúde, uma vez que exige investimento em recursos físicos e humanos, capacitação dos profissionais e aumento do fornecimento de serviços e políticas públicas ${ }^{(14)}$.

O número de pessoas não alfabetizadas, nessa pesquisa, foi expressivo assim como a idade entre 68 e 101 anos, na presença desses achados é possível comparar com o cenário nacional. De acordo com o censo de 2015 do Instituto Brasileiro de Geografia e Estatística (IBGE), a população com idade acima de 65 anos apresenta a maior taxa de não alfabetizados no Brasil. O baixo grau de escolaridade influencia negativamente o processo saúde doença, porque pode comprometer o acesso à educação em saúde, dificuldade na mudança dos hábitos de vida e controle dos fatores de risco ${ }^{(14-16)}$. Isto posto, é necessário compreender que o serviço e os profissionais podem encontrar dificuldades com relação ao processo de adesão terapêutica de vítimas com baixo grau de escolaridade, necessitando desenvolver estratégias acessíveis a esse público.

Em um estudo realizado no estado de Pernambuco, observou-se que a maioria dos usuários atendidos nos serviços de urgências possui renda entre um a três salários mínimos total de 99 (83,2\%), já no presente estudo, a renda mensal de um a dois salários mínimos obteve o número mais expressivo. Quanto à ocupação, o mesmo estudo apresentou que 42 (35,3\%) eram desempregados, o que difere com a presente pesquisa, na qual a maioria era do lar ou aposentado(a). Portanto, a condição econômica desfavorável pode influenciar de modo negativo a recuperação desses pacientes, uma vez que o alto custo com a terapia medicamentosa cria uma barreira para adquirir os medicamentos, comprometendo a melhora do paciente e aumentando a possibilidade de um novo episódio. Na literatura, não se encontrou trabalhos em que houvesse uma relação direta da renda e ocupação dos usuários com afecção neurológica e o SAMU, por este motivo, considerou no cenário geral dos atendimentos em serviço de urgência ${ }^{(17)}$.

A crise convulsiva é um problema frequente no serviço de urgência e emergência, e sua etiologia pode ser devido a diversos fatores, como por exemplo: distúrbios hidroeletrolíticos, intoxicação, abstinência de drogas, sedativos ou lesão neurológica. Após análise através de exames mais complexos, é possível identificar a causa desse evento. Portanto, durante essa pesquisa não foi possível descobrir a causa dessa manifestação clínica. Somado a esse fato, a literatura aborda em número maior a relação da etiologia que levou a crise convulsiva. Mas um estudo revelou que o atraso no diagnóstico não interfere com o tratamento e estabilização da vítima, pelo serviço de emergência. Diferentemente dessa pesquisa, a crise convulsiva expressou um número maior de ocorrências neurológicas em outro estudo realizado em Botucatu-SP(18-20). 
O Acidente Vascular Encefálico é dividido em isquêmico e hemorrágico, de acordo com o seu aspecto patológico. A terapia indicada em casos de AVE isquêmico é a trombólise através do ativador do plasminogênio tecidual recombinante (et-PA), no entanto essa terapia é vantajosa e segura quando o tratamento é iniciado em até 4,5 horas do início do quadro e após uma avaliação completa com exames de imagem, laboratoriais e excluir algum tipo de hemorragia. A consequência disto, na realidade, os pacientes acabam perdendo o tempo limite para início da terapia e, passando do tempo estimado, os estudos mostraram que aumentam os riscos de hemorragia e mortalidade ${ }^{(18,21-22)}$.

Por conseguinte, a presente pesquisa mostrou que as vítimas de AVE totalizaram um número expressivo de atendimentos pelo SAMU 192 RN. Convém inferir a necessidade do reconhecimento rápido dos sintomas desse agravo pelo serviço pré-hospitalar, a fim de diminuir o tempo de transporte do paciente e minimizar os riscos.

Este estudo identificou uma média de tempo resposta de 31 minutos para todas as afecções neurológicas, no entanto percebe-se que o tempo é fator determinante para minimizar danos e aumentar a sobrevida dessas vítimas ${ }^{(23)}$.

Além disso, estudos apontam que tempo resposta inferior a 10 minutos é o ideal nos serviços de atendimento pré-hospitalar. O tempo resposta é um dos indicadores que elevam a qualidade da assistência prestada ${ }^{(23-24)}$.

Diante da real importância do AVE, o presente estudo mostrou o perfil das pessoas vítimas dessa doença, sendo a maioria do sexo masculino, com idade entre 68 a 101 anos, corroborando com estudo realizado na Dinamarca, no qual a maioria eram do sexo masculino 58\%, com idade média de 66 anos. A variável ocupação identificou que a frequência maior era do lar ou aposentado(a), como também a pesquisa realizada no Ceará-CE mostrando que 54 (59,3\%) das vítimas de AVE eram aposentados. Já o grau de escolaridade, segundo um estudo realizado em Cajazeiras-PB, 19 (50\%) não possuíam estudo formal, achados semelhantes a essa pesquisa ${ }^{(25-27)}$.

Levando em consideração as lombalgias associadas a lesão medular, foram predominantes no sexo feminino, quanto à ocupação nenhum dos pacientes estavam desempregados, trabalhavam com construção civil e no setor de comércio e outros serviços. Achado semelhante foi observado em uma pesquisa realizada nos EUA, a maioria também eram mulheres e trabalhavam em tempo integral(28).

O turno de chamados para o SAMU 192 RN, nessa pesquisa, foi predominante pela manhã, já em outro estudo realizado no sul do país revelou que o turno das $12 \mathrm{~h} 00$ às $17 \mathrm{~h} 59 \mathrm{~min}$ totalizou $39 \%$ dos chamados. Em seguida, apareceu o turno da tarde sendo o segundo horário com maior chamado nesse estudo, divergindo de outro estudo que esse período há uma maior ocorrência de atendimentos ${ }^{(29,11)}$.

Quanto ao tipo de veículo utilizado, a Unidade de Suporte Básico (USB) atendeu a maioria dos chamados, dados semelhantes foram identificados no estudo realizado no mesmo estado, no qual 1319 $(41,4 \%)$ dos chamados foram socorridos pela USB. Outro dado que merece destaque é que a Unidade de Suporte Avançado (USA) foi utilizada na maioria dos chamados a caso de AVE. Pode-se inferir que esse fato deva-se a maior risco de complicação durante o trajeto desse paciente, necessitando de um serviço especializado ${ }^{(11)}$.

Com relação às limitações do estudo, foi observada a impossibilidade de analisar o tempo de transporte do local do evento até o serviço especializado e a periodicidade de ocorrência desse evento.

\section{- CONCLUSÃo}

O perfil epidemiológico identificado foi de homens idosos, que sofreram Acidente Vascular Encefálico, com déficit de escolaridade e renda, não receberam atendimento no tempo resposta adequado e foram transportados por Unidade de Suporte Básico.

A caracterização do perfil dos usuários atendidos pode subsidiar a elaboração de estratégias para enfrentamento desses agravos e melhoria da qualidade da assistência prestada pelos serviços préhospitalares móveis de urgência. Além disso, sugerem-se novos estudos dessa natureza e com maior periodicidade. 
1. Ministério da Saúde (BR). Secretaria de Atenção à Saúde. Departamento de Atenção Especializada. Manual instrutivo da Rede de Atenção às Urgências e Emergências no Sistema Único de Saúde (SUS). Brasília: Ministério da Saúde; 2013.

2. Duarte EC, Barreto SM. Transição demográfica e epidemiológica: a Epidemiologia e Serviços de Saúde revisita e atualiza o tema. Epidemiol. Serv. Saúde. [Internet] 2012;21(4) [acesso em 10 out 2016]. Disponível: http://dx.doi. org/10.5123/S1679-49742012000400001.

3. Ministério da Saúde (BR). Política nacional de atenção às urgências. Brasília: Ministério da Saúde; 2003.

4. Ministério da Saúde (BR). Portal da Saúde. [Internet] O que é o SAMU 192? [acesso em 06 set 2016]. Disponível: http://portalsaude.saude.gov.br/index.php/o-ministerio/principal/secretarias/951-sas-raiz/dahu-raiz/forcanacional-do-sus/l2-forca-nacional-do-sus/13407-servico-deatendimen to-movel-de-urgencia-samu-192.

5. National Hospital Ambulatory Medical Care Survey: 2011 Emergency Department Summary Tables. Natl Health Stat Report. [Internet] 2011 [acessoem 6 set 2016]. Disponível: https://www.cdc.gov/nchs/data/ahcd/nhamcs_ emergency/2011_ed_web_tables.pdf.

6. Lange MC, Braatz VL, Tomiyoshi C, Nóvak FM, Fernandes AF, Zamproni LN, et al. Neurological diagnoses in the emergency room: differences between younger and older patients.Arq Neuropsiquiatr. [Internet] 2011;69(2A) [acesso em 6 set 2016].Disponível:http://dx.doi.org/10.1590/S0004-282X2011000200014.

7. Hansen CK, Fisher J, Joyce N, Edlow JA. Emergency department consultations for patients with neurological emergencies. Eur J Neurol. [Internet] 2011;18(11) [acesso em 5 set 2016]. Disponível: http://dx.doi.org/10.1111/ j.1468-1331.2011.03390.x.

8. Barbetta PA. Estatística aplicada às ciências sociais. $8^{\mathrm{a}}$ ed. Florianópolis: Editora da UFSC; 2013.

9. Ministério da Saúde (BR). Conselho Nacional de Saúde. Diretrizes e normas regulamentadoras de pesquisa envolvendo seres humanos. Resolução n. 466, de 12 de dezembro de 2012. Brasília; 2012

10. Dantas RAN, Costa IKF, da Nóbrega WG, Dantas DV, Costa IKF, Torres GV. Ocorrências realizadas pelo serviço de atendimento móvel de urgência metropolitano. Rev enferm UFPE on line. [Internet]2014;8(4) [acesso em 6 set 2016]. Disponível: https://periodicos.ufpe.br/revistas/revistaenfermagem/article/viewFile/9751/9867.

11. Dias JMC, de Lima MSM, Dantas RAN, Costa IKF, Leite JEL, Dantas DV. Perfil de atendimento do serviço préhospitalar móvel de urgência estadual. Cogitare Enferm. [Internet] 2016;21(1) [acesso em 25 out 2016]. Disponível: http://dx.doi.org/10.5380/ce.v21i1.42470.

12. Machin R, Couto MT, da Silva GSN, Schraiber LB, Gomes R, Figueiredo WS, et al. Concepções de gênero, masculinidade e cuidados em saúde: estudo com profissionais de saúde da atenção primária. Ciênc. saúde coletiva. [Internet] 2011;16(11) [acesso em 15 nov 2016]. Disponível: http://dx.doi.org/10.1590/S1413-81232011001200023.

13. Marques GQ, Lima MADS, Ciconet RM. Agravos clínicos atendidos pelo Serviço de Atendimento Móvel de Urgência (SAMU) de Porto Alegre - RS. Acta paul.enferm. [Internet] 2011;24(2) [acesso em 24 out 2016]. Disponível: http://dx.doi.org/10.1590/S0103-21002011000200005.

14. Instituto Brasileiro de Geografia e Estatística (IBGE). Síntese de indicadores sociais: uma análise das condições de vida da população brasileira: 2015. Coordenação de População e Indicadores Sociais. Rio de Janeiro: IBGE; 2015.

15. Geib LTC. Determinantes sociais da saúde do idoso. Ciênc. saúde coletiva. [Internet] 2012;17(1) [acesso em 23 out 2016]. Disponível:http://dx.doi.org/10.1590/S1413-81232012000100015.

16. Malta DC,de Morais Neto OL,da Silva Junior JB. Apresentação do plano de ações estratégicas para o enfrentamento das doenças crônicas não transmissíveis no Brasil, 2011 a 2022. Epidemiol. Serv. Saúde. [Internet] 2011;20(4) [acesso em 28 out 2016]. Disponível:http://dx.doi.org/10.5123/S1679-49742011000400002.

17. de Oliveira ANS, Lima KSB F, Moura LA, Mendes RNC, Gomes JO, Moura JG. O perfil clínico epidemiológico dos usuários da rede de urgências no interior de Pernambuco. R. pesq. cuidado fundam. [Internet] 2013;5(2) 
[acesso em 27 out 2016]. Disponível: http://dx.doi.org/10.9789/2175-5361.2013.v5i2.3601-3607.

18. Martins HS, Neto RAB, Velasco IT. Medicina de emergência: abordagem prática. $11^{a}$ ed. São Paulo: Manole; 2016.

19. Claassen J,Riviello Jr JJ, Silbergleit R. Emergency neurological life support: status epilepticus. Neurocrit Care. [Internet] 2015;23(Suppl 2) [acesso em 16 nov 2016. Disponível:http://dx.doi.org/10.1007/s12028-015-0172-3.

20. de Almeida PMV, Dell'Acqua MCQ, Cyrino CMS, Juliani CMCM, Palhares VC, Pavelqueires S. Análise dos atendimentos do SAMU 192: Componente móvel da rede de atenção às urgências e emergências. Esc. Anna Nery. [Internet] 2016;20(2) [acesso em 10 out 2016]. Disponível:http://dx.doi.org/10.5935/1414-8145.20160039.

21. Ragoschke-Schumm A, Walter S, Haass A, Balucani C, Lesmeister M, Nasreldein A, et al. Translation of the 'time is brain' concept into clinical practice: focus on prehospital stroke management. Int J Stroke. [Internet] 2014;9(3) [acessoem 25 out 2016]. Disponível: http://dx.doi.org/10.1111/ijs.12252.

22. Ahmed N, Wahlgren N, Grond M, Hennerici M, Lees KR, Mikulik R, et al. Implementation and outcome of thrombolysis with alteplase 3-4•5 h after an acute stroke: an updated analysis from SITS-ISTR. Lancet Neurol. [Internet] 2010;9(9) [acesso em 25 out 2016]. Disponível: http://dx.doi.org/10.1016/S1474-4422(10)70165-4.

23. El Sayed MJ. Measuring quality in emergency medical services: a review of clinical performance indicators. Emerg Med Int. [Internet] 2012;(2012) [acesso em 31 mar 2017]. Disponível: http://dx.doi.org/10.1155/2012/161630.

24. David G, Harrington SE. The Quality of emergency medical services. LDI Issue Brief. [Internet] 2011;17(3) [acesso em 31 mar 2017]. Disponível: http://repository.upenn.edu/cgi/viewcontent.cgi?article=1017\&context=ldi issuebriefs.

25. SimonsenSA, Andresen M, Michelsen L, Viereck S, Lippert FK, Iversen HK. Evaluation of pre-hospital transport time of stroke patients to thrombolytic treatment. Scand J Trauma Resusc Emerg Med. [Internet] 2014;(22) [acesso em 25 out 2016]. Disponível: http://dx.doi.org/10.1186/s13049-014-0065-z.

26. Cavalcante TF, de Araújo TL, Moreira RP, de Santiago JMV. Perfil socioeconômico de pacientes internados por acidente vascular encefálico. Rev. Rene. [Internet] 2010;11(4) [acesso em 24 out 2016]. Disponível: http://www. revistarene.ufc.br/vol11n4_pdf/a17v11n4.pdf.

27. Lopes Junior JEG, de Freitas Júnior JHA, de Figueiredo ADJ, de Santana FM. Perfil dos pacientes acometidos por Acidente Vascular Encefálico cadastrados na Estratégia de Saúde da Família. Rev Fisioter S Fun. [Internet] 2013;2(1) [acesso em 29 out 2016]. Disponível: http://www.fisioterapiaesaudefuncional.ufc.br/index.php/ fisioterapia/article/view/193.

28. MehlingWE, Gopisetty V, Bartmess E, Acree M, Pressman A, Goldberg H, et al. The prognosis of acute low back pain in primary care in the United States: a 2-year prospective cohort study. Spine (Phila Pa 1976). [Internet] 2012;37(8) [acesso em 26 out 2016]. Disponível: http://dx.doi.org/10.1097/BRS.0b013e318230ab20.

29. Casagrande D, Stamm B, Leite MT. Perfil dos atendimentos realizados por uma Unidade de Suporte Avançado do Serviço de Atendimento Móvel de Urgência (SAMU) do Rio Grande do Sul. Scientia Medica. [Internet] 2013;23(3) [acesso em 16 out 2016]. Disponível: http://revistaseletronicas.pucrs.br/ojs/index.php/scientiamedica/ article/download/13343/10204 